\title{
Futuro de los profesionales de la información: nuevos productos conllevan nuevos roles
}

\author{
Por Bernabé Zea
}

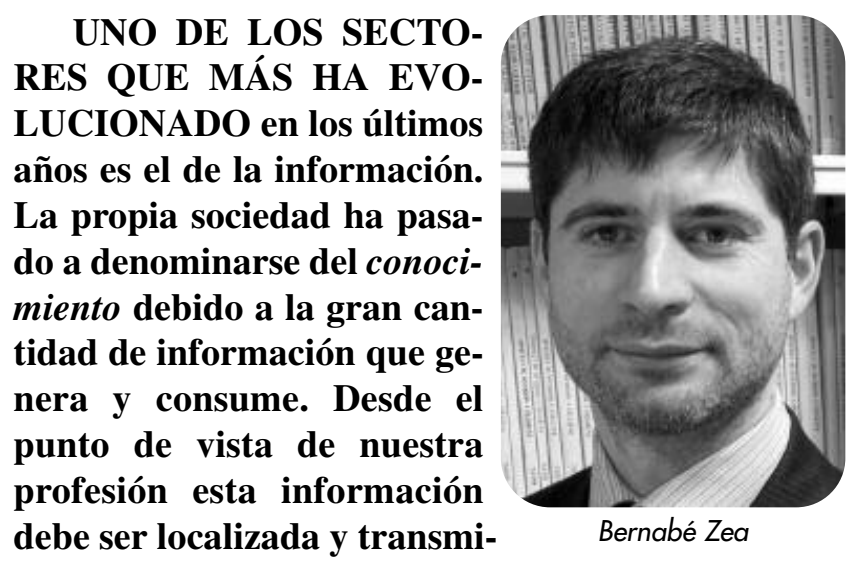

tida de forma conveniente para que sea útil. Las empresas del sector han crecido creando nuevos y más potentes productos para satisfacer la demanda existente. En estas circunstancias, y como no podía ser de otro modo, el profesional de la información tiene grandes oportunidades, pero también grandes retos.

Ha habido tres grandes hechos que han cambiado su entorno:

— generalización del documento electrónico, que ha facilitado mucho a los usuarios finales el acceder directamente al documento original;

—disponibilidad de interfaces simples, e incluso con cierta inteligencia en la elaboración e interpretación de las consultas, que permiten su utilización a usuarios sin apenas formación previa;

—eclosión de internet como un vehículo para el transporte de la información.

Antes de continuar es importante reseñar que la importancia de internet en el sector de la documentación no ha sido tanto por la aparición de nuevos contenidos, ya que los mismos existían con anterioridad (revistas, patentes y grandes bases de datos comerciales), sino como medio para la distribución de dicha información alternativo a los servicios en línea clásicos (como Dialog o STN) o las propias bibliotecas. La información directamente generada en internet se produce sin controles que puedan contrastar su calidad y, por lo tanto, tiene un interés relativo en sectores económicamente importantes, como son la ciencia y la tecnología, y, sobre todo, las patentes. El hecho de que la gran mayoría de búsquedas que se llevan a cabo en los servicios en línea estén directamente relacionadas con las patentes ilustra la importancia económica de estas últimas.

\author{
"Quien mejor sabe la \\ información que busca es el \\ usuario final, el propio \\ interesado"
}

La conjunción de los tres hechos antes comentados ha supuesto una auténtica revolución, pero más para el usuario final que para el profesional de la información. Durante los últimos diez años se han lanzado por primera vez productos electrónicos capaces de ser utilizados directamente por el usuario final de forma simple, sin apenas aprendizaje, y en muchas ocasiones permitiéndole obtener mejores resultados que los que previamente podía conseguir a través de un documentalista profesional. Esta última aseveración posiblemente no sea fácil de aceptar por algunos profesionales. Sin embargo me parece indiscutible que la gran cantidad de información especializada existente exige tener un conocimiento real de la materia a buscar (química, ingeniería, patentes, negocios, etc.) para poder recuperar y seleccionar la información que precisa el usuario. Lamentablemente, el profesional de la información no siempre ha tenido la formación que le permita dicho conocimiento; a veces incluso es prácticamente imposible que esté capacitado para interpretar los resultados del estado del arte.

\section{"Cada vez más el usuario final tiene más facilidades y más transparencia de operación para obtener la información por sí mismo"}

Por otro lado, el aumento de las prestaciones informáticas ha permitido la integración de los sistemas de búsqueda con los propios documentos completos. Es más, de la integración se está pasando a la fusión de ambos. Las bases de datos de texto completo se están multiplicando, ya que apenas necesitan trabajo de edición y que el coste informático para su elaboración es 


\title{
Próximos temas centrales
}

\author{
Enero 2007 Ciencia y tecnología de la información (InSciT2006) \\ Marzo 2007 Web 2.0: blogs, participación y Lib $\mathbf{2 . 0}$ \\ Mayo 2007 Portales y gestión de contenidos (CMS)
}

Julio 2007 Bibliotecas universitarias

Septiembre 2007 Documentación multimedia

Noviembre 2007 Ontologías

Los interesados pueden remitir notas, artículos, propuestas, publicidad, comentarios, etc., sobre estos temas a:

epi@elprofesionaldelainformacion.com

cada vez menor. Este mismo hecho puede explicar la adición de texto de los documentos originales a la base de datos bibliográfica World patents index (WPI). De nuevo las patentes liderarán este hecho por su interés económico y porque estos documentos, al ser publicaciones oficiales, generalmente no están sujetos a derechos de autor, con lo que todos los proveedores de servicios de información pueden utilizar libremente su contenido. Así, es muy posible que otras grandes bases de datos de patentes distintas de WPI integren su propia indexación, que es su principal valor añadido, con el texto completo de las patentes, y quizás también con la situación legal de las mismas. El resultado final serían productos que hasta hace unos años eran sólo un sueño para los documentalistas especializados en patentes.

\section{"El profesional de la información puede dedicarse ahora a realizar tareas de mayor valor añadido y más prestigio dentro de su organización"}

Ante la aparición de servicios como SciFinder, Web of knowledge, Science direct, Scopus o Integrity (producido por la editorial catalana Prous Science), que integran la búsqueda con la visualización de los documentos completos; de nuevas herramientas de análisis y visualización de los resultados de las búsquedas, tales como STN AnaVist, las cuales permiten el análisis de la información recuperada para la toma de las decisiones estratégicas de la empresa; y de otros productos que están por llegar, el profesional de la información tiene dos grandes opciones, ambas válidas y reales en estos momentos, y que le permitirán gozar de un mayor reconocimiento laboral.

- La primera opción es dejar de ser un proveedor pasivo de información para convertirse en un miembro activo del equipo de toma de decisiones estratégicas en la relación con la información de la empresa. De esta forma, no sólo generaría información, sino que además se convertiría en miembro de un equipo que debe integrar la información de la empresa. Normalmente esta persona dejaría de depender de la estructura de la biblioteca y pasaría a tener una posición de mayor responsabilidad, convirtiéndose en directivo de la información.

Entre sus funciones se encontraría la evaluación de los productos existentes en el mercado; la negociación (tarifas planas y otras opciones) y la adquisición de aquéllos que son de mayor interés para las necesidades de sus usuarios; la integración de los productos adquiridos con los ya existentes, por ejemplo con la implementación de sistemas de búsquedas federadas; la formación de sus usuarios (en algunos casos haciendo simplemente de intermediario con los instructores de la empresas que proporcionan los productos), etc. En mi opinión, todavía tendrían una última función importante: ser capaces de determinar el beneficio que dichas herramientas ofrecen a la organización.

En la actualidad las grandes empresas comienzan a llevar a cabo los denominados estudios del retorno de la inversión (return on investment, ROI) también sobre 
sus sistemas de información. Así, en el sector farmacéutico, uno de los que utilizan de forma más intensiva la información, se hacen estudios cuantitativos sobre qué ahorro se consigue por el uso de productos de información en aspectos tales como el incremento de la eficacia de la investigación o el abandono de líneas de investigación comercialmente improductivas (p. ej. líneas que se descubren patentadas por terceros y que, por tanto, no podrán ser protegidas ni explotadas). En un momento u otro el directivo de la información tendrá que estar familiarizados con este tipo de trabajos, yendo más allá del simple estudio de las estadísticas de uso.

\section{"El profesional de la información siempre estará por encima en cuanto a las fuentes y sus características, y en saber realizar las estrategias de búsqueda óptimas"}

- La otra gran opción del profesional de la información es la especialización en la búsqueda, de forma que sea capaz de dar al usuario final lo que éste no es capaz de conseguir con los proveedores a los que tiene acceso. No es discutible que los productos para el usuario final no tienen las prestaciones de los clásicos servicios en línea; pero tampoco lo es que para conseguir utilizar dichos servicios con todo su potencial se debe conocer a fondo, no sólo el lenguaje de recuperación y el contenido de las bases de datos, sino también, la materia que se pretende buscar y herramientas para la edición posterior de la información obtenida.

El perfil de este profesional no es fácil de encontrar, y especialmente hay España hay déficit de los mismos, principalmente en el sector químico-farmacéutico. Las continuas ofertas de trabajo que no pueden ser cubiertas adecuadamente son una prueba de ello. La falta de una formación adecuada es la primera de las causas, ya que ni las facultades de ciencias ni las de biblioteconomía-documentación tienen una opción académica adecuada para su formación. Por otro lado, la implementación de los servicios para usuarios finales en muchas empresas se ha producido antes de que existiera en las mismas un documentalista que hiciera las búsquedas o, si existía, se le ha desplazado a tareas de gestión que no le han posibilitado completar su formación como documentalista.

En resumen, con toda seguridad existe un buen futuro laboral como profesional de la información para quien se decida por cualquiera de las dos opciones indicadas. La primera de ellas supone tener un perfil de decisión y gestión que no era habitual hasta hace unos años entre los profesionales del sector. La segunda se restringe a los sectores de mayor interés comercial, como es la farmacia y, sobre todo, a todos los aspectos relativos a las patentes. En la práctica, debido a la necesidad de formación técnica, esta última opción se ha convertido en un coto privado de profesionales provenientes de estudios técnicos, en particular, químicos y profesionales cercanos.

\section{"Dominar el mundillo de la documentación de patentes garantiza encontrar un buen puesto de trabajo"}

Por último, creo indispensable aclarar que las dos opciones no son necesariamente incompatibles entre sí, sino que son perfectamente complementarias dentro de una empresa o institución. Es más, posiblemente las dos se potencien mutuamente. Así, un buen gestor de la información debería tener presente que el trabajo de un documentalista profesional no puede ser sustituido completamente por las búsquedas de los usuarios finales, y menos en los casos en que las decisiones derivadas de las mismas impliquen importantes repercusiones económicas. Por otro lado, si una institución o empresa tiene necesidad de un documentalista también tendrá necesidad de gestionar su información adecuadamente. O sea, que en algunos casos los dos roles podrán ser desempeñados por una misma persona, aunque lo normal es que no sea así, pues sus funciones se están alejando entre sí cada vez más.

Con la evolución actual de las empresas españolas hacia las nuevas tecnologías y la inversión en I+D, el aumento de sus necesidades de información parece estar asegurado. Sin embargo, el tamaño de la empresa puede ser un factor limitante a la hora de disponer de buenos profesionales de la información. La subcontratación (outsourcing) puede convertirse en una buena opción para muchas pequeñas y medianas empresas, lo que debería abrir las puertas a nuevas oportunidades para los profesionales.

En definitiva, todo hace indicar que, afortunadamente, hay un futuro esperanzador para los profesionales de la información que sepan adaptarse a los nuevos roles que demanda el mercado laboral.

Bernabé Zea. Centro de Patentes de la Universitat de Barcelona

Representante para España de CAS/STN

Parc Científic de Barcelona, Baldiri Reixac, 4 08028 Barcelona

Tel.: +34-934034 511; fax: 934034517

bernabezea@pcb.ub.es

http://www.pcb.ub.es/centredepatents 